\title{
PREISACH MAPS OF MULTILAYERED Co/Cu STRUCTURES*
}

\author{
M. Gutowski, M.U. Gutowska, K. Piotrowski, L.T. Baczewski \\ AND A. WAWRO \\ Institute of Physics, Polish Academy of Sciences \\ Al. Lotników 32/46, 02-668 Warszawa, Poland
}

Magnetooptical Kerr effect measurements of room temperature hysteresis loops were taken using sandwich- and multilayer-type specimens and $\mathrm{He}-\mathrm{Ne}$ laser light. For maximal external field of $\sim 250$ Gs the corresponding Kerr angle reached $0.04 \mathrm{deg}$. The samples were obtained in the Institute of Physics, Polish Academy of Sciences, Warszawa, using MBE method. The structure of samples may be described by a formula: substrate-buffer layer- $(x \mathrm{Co} / y \mathrm{Cu})_{n}$-cover layer. $\mathrm{Al}_{2} \mathrm{O}_{3}$ (two orientations) and $\mathrm{MgO}$ were used as substrates, the buffer layer was made of $\mathrm{W}, \mathrm{Cu}$ or $\mathrm{Fe}, x=15,20$ or $25 \AA, y=7,8,9,10,11,12,13,14$, and $20 \AA, n=25$ and $30.50 \AA$ of gold (Au) served as a cover layer. The genetic algorithm was subsequently used as a data processing tool, in order to reconstruct the Preisach map for each hysteresis loop. The diagrams clearly indicate changes of magnetic interactions caused by varying thicknesses of individual magnetic and non-magnetic layers.

PACS numbers: $75.40 . \mathrm{Mg}, 75.60 . \mathrm{Ej}, 75.70 .-\mathrm{i}, 78.20 . \mathrm{Ls}, 78.66 .-\mathrm{w}$

\section{Introduction}

The idea of finding an analytical formula describing the hysteresis loop for magnetic materials is rather old. The elegant solution, first proposed by Preisach [1] in 1935, was not immediately put into practice due to the lack of satisfactory computing power available then. It was relatively easy to simulate the hysteresis curve having the Preisach map, but the inverse was (and still is) rather difficult. Only in 1950's first more or less successful attempts to solve this problem were reported [2-4]. After 1960 more papers dealing with related subjects appeared, almost all of them proposed various improvements and extensions of original Preisach's idea.

In this paper we present Preisach maps obtained by a genetic algorithm and using only one simple a priori assumption concerning the Preisach density. The samples under study have a layered structure, with a varying spacing between magnetic layers, so the resulting diagrams are quite interesting. It is, of course, a matter of interpretation, which is still under investigation [5], what can be seen on Preisach diagrams.

*This work was supported in part by the Committee for Scientific Research grant No. 8T11B 05309. 


\section{Experimental setup}

There are three basic experimental configurations to investigate the rotation of polarization plane of light reflected by the magnetized sample. In this study the so-called longitudinal effect was investigated. In the Cartesian coordinate system the laser light travels in $x z$ plane $(k \perp y)$, while the sample plane is $x y$. The vector of external magnetic field $\boldsymbol{H}$, produced by a coil, and magnetization of the sample, $M$, are parallel to $x$ axis. The magnitude of rotation is strictly proportional to the magnetization on the surface of the sample and up to $200 \AA$ below. Only the component of $\boldsymbol{E}$, which is perpendicular to the sample plane, is rotated. The Kerr constant, describing the magnitude of effect, depends on a reflection angle, and, for many materials, achieves a maximal value when the angle between $k$ and $z$ is close to 60 degrees. In our setup this angle is limited by the coil geometry to $\sim 75$ degrees. All the measurements were taken at room temperature. Two examples are shown in Fig. $1 \mathrm{~d}$ and $\mathbf{f}$.

\section{The Preisach map}

In the Preisach [1] model, the magnetic body is assumed to consist of a number of hysterons. The interpretation of hysterons is still unclear (they are not magnetic domains); they are fully characterized by their reaction to magnetic field. Each hysteron has two distinct states corresponding to its magnetic saturation, either in the direction parallel to the external magnetic field or anti-parallel. The switching from one state to another occurs at two well-defined values of external magnetic field, $a$ and $b$, i.e. at two switching fields. The summary of hysteron's behavior is given in Table. Each hysteron represents a single point on a plane $a b$, called therefore the Preisach plane. The complete distribution of hysterons, $\rho=\rho(a, b)$ (Preisach density), describing the magnetic behavior of a given sample, is called the Preisach map or Preisach diagram.

\section{TABLE}

Behavior of hysteron in an external magnetic field, $H$.

\begin{tabular}{l|l}
\hline \hline Magnetic field value, $\bar{H}$ & State of hysteron \\
\hline$H<b$ & $-M_{\mathrm{s}}$ (-saturation) \\
$b<H<a$ & $?$ (depending on previous history) \\
$\qquad a<H$ & $+M_{\mathrm{s}}$ (+saturation)
\end{tabular}

Since the field $a$, causing switching to + saturation, always exceeds the field $b$ (switching to -saturation), and there are no hysterons outside the rectangle $\left(|a| \leq H_{\max }\right) \times\left(|b| \leq H_{\max }\right)$ where the field $H_{\max }$ is the one, which saturates the sample, then the support of the distribution $\rho$ forms a triangle on the Preisach plane.

Knowledge of the distribution $\rho$ makes it possible to reproduce the magnetic response of a sample to the arbitrary sequence of external fields, i.e. both major and minor hysteresis loops. $\rho$ is normalized in such a way that $\iint \rho(a, b) \mathrm{d} a \mathrm{~d} b=$ $2 M_{\mathbf{s}}$. The correspondence between the features found on the Preisach maps and microscopic parameters of the samples has not been established yet. 


\section{Calculations}

A reconstruction of the Preisach maps was done using only the hysteresis data obtained experimentally. A single a priori assumption was made concerning the symmetry of the searched distribution, $\rho(a, b)=\rho(-b,-a)$, which is valid for samples of macroscopic sizes. The method used was the smooth genetic algorithm [6], implemented for this two-dimensional case. This is essentially one of the Monte-Carlo-type optimization methods. The objective function was chosen as a sum of absolute values of differences between simulated and measured values on the hysteresis curve weighted by dispersions of individual measurements. Each experimental hysteresis loop consisted of $72-522$ measurements, typically 220 .

\section{Results and conclusions}

Figure 1 shows a few examples of many Preisach maps we have obtained for various combinations of magnetic layer and non-magnetic spacers' thicknesses. It

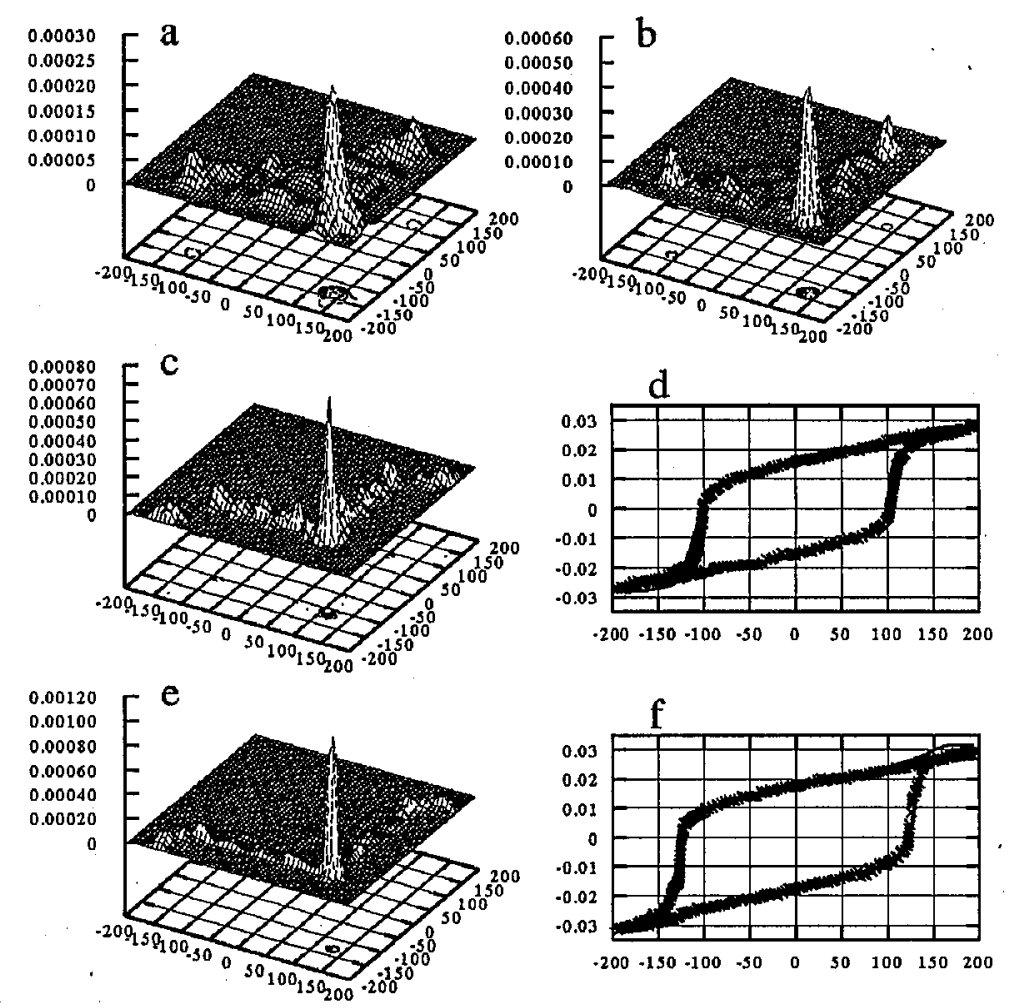

Fig. 1. Preisach maps for samples consisting of 30 layers of Co (15 $\AA$ thick) interleaved with $\mathrm{Cu}$ layers with thicknesses $13 \AA$ (a), $11 \AA$ (b), $10 \AA$ (c) and $9 \AA$ (e). Note a change of the position of the highest peak on maps. Hysteresis loops $(d, f)$ correspond to maps (c, e), respectively. The experimental points and simulated curve are shown. Kerr rotation angles are expressed in degrees, Preisach density in $\mathrm{Oe}^{-2}$, all other quantities are magnetic field strength, in Oe. 
is readily seen that thick layers of Co behave almost like the bulk material when the spacing between them is small ( $9 \AA$, picture (e)). A very similar map (not shown) was obtained when the thickness of Cu interlayers exceeded $20 \AA$. The difference is that in the latter case there are no visible traces of magnetic interactions between individual layers, i.e. there is only one single peak on the Preisach map, located on the symmetry axis. When the distance between interacting Co layers increases then the landscape becomes more and more complicated (pictures (c), (b), and (a)). Since the material under investigation is metallic, then we should expect, that at least some exchange integrals may have different signs (oscillating RKKY interactions). The presence of hills located far from the symmetry axis $b=-a$ seems to support this hypothesis rather well. The features present on the line $b=a$ correspond to the small isolated magnetic clusters or even single Co ions, which behave as (super)paramagnetic particles exhibiting no hysteresis at all. The intensity of those details may be used to judge the quality of samples (interface roughness). The resolution on the maps is $\sim 28 \mathrm{Gs}$; this is the reason for the systematic deviations between simulated hysteresis curves and experimental points. This effect is especially easy to spot on the part (d) of Fig. 1.

\section{References}

[1] F. Preisach, Z. Phys. 94, 277 (1935).

[2] L. Néel, Appl. Sci. Res. B 4, 13 (1954).

[3] G. Biorci, D. Pescetti, Nuovo Cimento 7, 829 (1958).

[4] G. Biorci, D. Pescetti, J. Appl. Phys. 37, 425 (1966).

[5] D.J. Dunlop, M.F. Westcott-Lewis, M.E. Bailey, Phys. Earth Planet. Inter. 65, $62(1990)$.

[6] M. Gutowski, J. Phys. A 27, 7893 (1994). 